\title{
ANÁLISIS DEL ÍNDICE GENERAL DE LA BOLSA DE VALORES DE COLOMBIA Y SUS RENDIMIENTOS DESDE LA TEORÍA DEL CAOS, 2001-2011*
}

\author{
Hermilson Velásquez Ceballos ${ }^{* *}$ \\ Jorge Humberto Restrepo Restrepo ${ }^{* * *}$
}

Recibido: julio 21 de 2011

Aceptado: junio 08 de 2012

\section{RESUMEN}

El objetivo de este trabajo es presentar un enfoque alternativo para el análisis de las series de tiempo en mercados financieros, cuyos fundamentos consideran la posible existencia de características de objetos fractales y estructuras caóticas en ellas, lo cual permite diseñar estrategias de negociación que tengan en cuenta esta información. El procedimiento que se siguió para el contraste de la hipótesis requirió validar: no-linealidad, no-normalidad, auto similitud, persistencia, y caos. El esquema propuesto se aplica a las series del índice general de la Bolsa de Valores de Colombia (IGBC) y a sus rendimientos, en el período comprendido entre julio de 2001 y mayo de 2011 . Se encontró evidencia de caos y de comportamiento fractal en las series analizadas, y una de las consecuencias directas es la posibilidad de diseñar estrategias alternativas de negociación que podrían ser aprovechadas en procesos de transacciones en la Bolsa de Valores de Colombia.

\section{PALABRAS CLAVE:}

Mercados fractales, autosimilitud, persistencia, caos, índice bursátil.

\section{CLASIFICACIÓN JEL}

\author{
G14, C40
}

\section{CONTENIDO}

Introducción; 1. Metodología; 2. Datos; 3. Resultados; 4.Conclusiones; Bibliografía; Anexos.

\footnotetext{
Artículo de investigación, es uno de los resultados obtenidos por Jorge Restrepo Restrepo de su tesis de Maestría en Finanzas de la Universidad EAFIT bajo la asesoría del profesor Hermilson Velásquez Ceballos, profesor de la Escuela de Economía y Finanzas e integrante de los grupos de investigación en Finanzas y Banca clasificado en Colciencias en la categoría B y del grupo Research in Spatial Economics(Rise) de la Universidad EAFIT.

** Licenciado en Matemáticas, Universidad del Quindío, Armenia, Colombia. Magíster en Matemáticas Aplicadas Universidad EAFIT, Medellín, Colombia. Doctor en Ciencias Matemáticas, Universidad Politécnica de Valencia. Valencia, España. Docente e investigador, Escuela de Economía y Finanzas, Universidad EAFIT. Correo electrónico: evelas@eafit.edu.co.

.** Economista, Universidad de Cartagena. Cartagena, Colombia. Estudiante de la Maestría en Finanzas, Universidad EAFIT, Medellín, Colombia. Correo electrónico: jrestr86@eafit.edu.co.
} 


\title{
ANALYSIS OF THE COLOMBIAN STOCK EXCHANGE INDEX AND ITS YIELDS SEEN FROM THE CHAOS THEORY 2001-2011
}

\section{ABSTRACT}

The objective of this article is to present an alternative for analyzing time series in financial markets, the foundations consider the possible existence of fractal objects and chaotic structure elements within them, thus enabling the design negotiation strategies that take this information into account. In order to test the hypothesis the following elements had to be validated: non-linearity, non-normality, self-similarity, persistency and chaos. The proposed scheme is applied to the series of the Colombian stock exchange index (IGBC) and its yields between July 2001 and May 2011. Evidence was found confirming the presence of chaos and fractal behavior in the analyzed series and one of the direct consequences is the possibility of designing alternative negotiation strategies that could be used in the trading process in the Colombian stock exchange.

\section{KEY WORDS}

Fractal markets, self-similarity, persistence, chaos, stock market index.

\section{JEL CLASSIFICATION}

\author{
G14, C40
}

\section{CONTENT}

Introduction; 1. Methodology; 2. Data; 3. Results; Conclusions; Bibliography, Attachments.

\section{ANALISE DO ÍNDICE GERAL DA BOLSA DE VALORES DA COLÔMBIA E SEUS RENDIMENTOS DESDE A TEORIA DO CAOS, 2001-2011}

\section{RESUMO}

O objetivo deste trabalho é apresentar um enfoque alternativo para a análise das series de tempo em mercados financeiros, onde os fundamentos consideram a possível existência de características de objetos fractais e estruturas caóticas nelas, o que permite desenhar estratégias de negociação que tenham em conta esta informação. O procedimento que se seguiu para o contraste da hipótese precisou validar: não linearidade, não normalidade, auto similitude, persistência, e caos. O esquema proposto se aplica às series do índice geral da bolsa de valores da Colômbia (IGBC) e aos seus rendimentos, no período compreendido entre julho de 2001 e maio de 2011. Encontrou-se evidencia de caos e de comportamento fractal nas series analisadas, e uma das conseqüências diretas é a possibilidade de desenhar estratégias alternativas de negociação que poderiam ser aproveitadas em processos de transações na bolsa de valores da Colômbia.

\section{PALAVRAS-CHAVE}

Mercados fractais, auto similitude, persistência, caos, índice bursatil

\section{CLASSIFICAÇÃO JEL}

\author{
G14, C40
}

\section{CONTEÚDO}

Introdução; 1. Metodologia; 2. Dados; 3. Resultados; 4.Concluções; Bibliografia; Anexos. 


\section{INTRODUCCION}

El estudio de la dinámica para índices y rendimientos en los mercados de valores resulta fundamental para la comprensión del desempeño de los mercados financieros. La mayoría de los modelos para el análisis del comportamiento de estos mercados y los precios de las acciones se basan en la Hipótesis de los Mercados Eficientes (HME), introducida por Eugen Fama (Fama y otros, 1969), que supone que los precios de equilibrio del mercado reflejan toda la información nueva e histórica de forma inmediata, y los agentes del mercado se comportan de manera racional y con aversión al riesgo. Desde entonces este ha sido el paradigma dominante.

En los años recientes se ha propuesto una alternativa de investigación fundamentada en las Hipótesis de los Mercados Fractales (HMF), introducida por Peters (1991), que utiliza geometría fractal y teoría del caos, para describir formas y procesos complejos por medio de reglas y algoritmos simples, que mediante su iteración se tornan complejas pero conservan su relación con el todo'; y combinados con métodos cuantitativos tradicionales permiten hacer una aproximación a la explicación de la dinámica del mercado, en el que se tienen en cuenta los movimientos diarios y eventos, como los desplomes de precios y el retiro del mercado de un número importante de agentes que transan en él.

La teoría del caos estudia los sistemas dinámicos sensibles a las variaciones de las condiciones iniciales. Este tipo de sistemas son determinísticos y pueden clasificarse como estables, inestables y caóticos. Los sistemas estables a lo largo del tiempo tienden a un punto u órbita de acuerdo con los atractores que los rigen; los sistemas inestables escapan a los atractores; y los sistemas caóticos, por la acción de los atractores, en unos momentos del tiempo son atraídos y en otros momentos son alejados; de esta manera se genera una dinámica deterministica.

El término fractal que proviene del latín "fractus" significa fragmentado; fue acuñado por Mandelbrot (1983), y se aplica a las formas geométricas generadas por procesos de repetición o iteración. Un fractal es una estructura que está compuesta por pequeñas partes que son similares entre sí y, a su vez, se parecen a la estructura completa; estas son las características que determinan lo que se denomina autosimilitud. Los fractales se pueden clasificar en lineales, complejos y caóticos, y resulta importante cuantificar la parte del espacio que ocupa, que se denomina dimensión fractal.

Bajo la HMF, el precio de transacción es siempre justo porque todos los agentes del mercado llegan a él con toda la información disponible, y reaccionan de manera similar ante la información recibida; pero la dinámica interna se establece por las

Propiedad de los fractales conocida como autosimilitud 
interacciones de los agentes, que pueden clasificarse en diferentes grupos de acuerdo con la forma como evalúan e incorporan la información en el proceso de toma de decisiones y, por tanto, es de esperar que grupos de agentes reaccionen de manera diferente ante un mismo estímulo. La utilización del enfoque fractal permite realizar análisis de los mercados con menos supuestos teóricos que otros modelos y, por lo tanto, los resultados bajo estos supuestos se pueden considerar más robustos (Casparri y Moreno, 2008).

Estudios realizados con el enfoque fractal para varios índices bursátiles de los principales mercados de capitales así como para acciones, tasas de interés y tasas de cambio, entre otras, han evidenciado la existencia de estructuras fractales, persistencia y memoria de largo plazo (Bartolozzi y otros, 2005; Nawrocki, 1995). En la literatura existen estudios de mercados bursátiles desde la perspectiva de la teoría del caos y los fractales, en cuatro mercados latinoamericanos (Argentina, Brasil, Chile y México); resultados similares se encuentran para los mercados de Grecia y Turquía (Özer y Ertokatli, 2010; Ozun, Hanias y Curtis, 2010).

Presentar alternativas de análisis para los mercados financieros fundamentadas de manera formal con rigurosidad científica y que puedan ser implementadas computacionalmente se constituye en líneas de trabajo que deben ser estudiadas. El presente trabajo utiliza un enfoque con soporte matemático, estadístico y de series de tiempo para identificar la presencia de elementos característicos de los fractales y de estructuras caóticas en las series del Indice General de la Bolsa de Colombia y de los rendimientos, con información comprendida en el período de tiempo desde la fusión de las tres bolsas de valores que existían en Colombia en el año 2001 (Bolsa de Valores de Bogotá, Bolsa de Valores de Medellín y Bolsa de Valores de Cali) cuando se crea el Índice General de la Bolsa de Valores de Colombia hasta el momento de la creación de lo que se ha denominado Mercado Integrado Latinoamericano (MILA) en junio de 2011.

Para las series objeto de estudio no se encuentra que no se cumple el supuesto de normalidad y linealidad; por tanto, existe la posibilidad de que estos sistemas sean caóticos. El procedimiento formal exige, además, realizar análisis de autosimilitud, persistencia y caos. El resultado central muestra evidencia a favor de la existencia de estructuras caóticas y de características fractales para las series analizadas, hecho que constituye el punto de partida para considerar otras estrategias de modelación, como son los modelos afirma y procesos determinísticos, entre los cuales se tienen las redes neuronales, alternativas que enriquecen el análisis para la toma de decisiones por parte de los agentes que participan en estos mercados.

Este trabajo está organizado de la siguiente forma: inicialmente se presentan los elementos centrales que se deben tener en cuenta para realizar estudios bajo este 
enfoque y que en este caso constituye la metodología, en la siguiente sección se hace una descripción de los datos, luego se presentan y se analizan los resultados. Al final se tienen las conclusiones, la bibliografía y los anexos.

\section{METODOLOGÍA}

El análisis que se utiliza para contrastar la posible existencia de estructuras caóticas y de fractal requieren de una presentación estructurada que oriente el proceso como se encuentra en Restrepo (2011). Los elementos centrales para realizar estos análisis lo constituyen: pruebas de normalidad, no linealidad, autosimilitud para la cual se utilizan métodos gráficos y métodos formales como la dimensión fractal y la dimensión de lagunaridad, persistencia que requiere el cálculo del exponente de Hurst, y para verificar la existencia de caos se utiliza el exponente de Lyapunov. En este artículo se hace un resumen de los elementos que se desarrollan en Restrepo (2011) y se presentan a continuación.

\subsection{Normalidad}

Los procesos caóticos tienen entre sus características, la ausencia de normalidad y la no linealidad; por tanto, el punto de partida para este análisis lo constituye la verificación de la normalidad en las series. Existen diferentes alternativas para realizar este contraste; en este trabajo se utilizan Jarque-Bera, Shapiro-Wilk, Shapiro-Francia.

\subsection{Linealidad}

La no linealidad de las series de datos se contrasta mediante la prueba BDS, propuesta por Brock y otros (1996) que se aplica sobre los residuos de un modelo ARIMA, filtro utilizado para cada una de las series analizadas; también se muestra evidencia de no linelalidad verificando que en estos procesos se tienen efectos del tipo ARCH.

\subsection{Autosimilitud}

Es una propiedad de los fractales en la que cada una de las partes tiene similitud con el todo; una aproximación a este fenómeno se realiza a partir de gráficos de las series con datos en diferentes frecuencias, para identificar la posible existencia de patrones y tendencias similares y se comprueba de manera formal por medio de la dimensión fractal y la dimensión de lagunaridad.

\subsection{Dimensión fractal y dimensión de lagunaridad}

Son indicadores de autosimilitud estadística; la dimensión fractal expresa la forma como un objeto llena el espacio que lo contiene. Esta medida siempre es 
positiva y para los objetos sólidos es un número entero; así, para un punto, es 0, para una línea es 1, para un plano completo acotado es 2 y para una esfera es 3. Dado que los fractales no alcanzan a llenar todo el espacio que los contiene su dimensión fractal es menor que la del espacio. Las series de tiempo no llenan de manera completa el plano que las contiene, por lo que su dimensión fractal está entre 1 y 2.

La dimensión fractal de una serie financiera brinda una visión más realista del riesgo que la varianza del activo (Casparri y Moreno, 2008); esto se debe a la no-normalidad que en general presenta la distribución de los activos financieros (Peters, 1991).

Lagunaridad $^{2}$ es el término que en geometría fractal se utiliza para determinar cómo un objeto fractal llena un espacio; es empleado para clasificar los fractales y el término se debe a Maldenbrot (1983). El valor del índice de lagunaridad se obtiene mediante un procedimiento iterativo de conteo del número de cajas; que existen en una cuadrícula, con recuadros de una longitud preestablecida y se conoce con

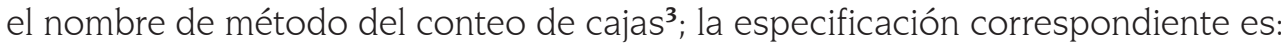

$$
\mathcal{L}(r)=\frac{\sum_{i=1}^{r^{2}} S^{2} Q\left(S_{i}, r\right)}{\left(\sum_{i=1}^{r^{2}} S^{2} Q\left(S_{i}, r\right)\right)^{2}}
$$

Donde, $\mathrm{S}_{i}$ es el número de puntos con información que llenan la caja $i, \mathrm{y} \mathrm{Q}\left(\mathrm{S}_{i}, r\right)$ es la distribución de frecuencia normalizada de $S_{i}$ para los diferentes tamaños de las cajas.

\subsection{Persistencia}

La persistencia de una serie está relacionada con la memoria de la serie y su capacidad de seguir tendencias a lo largo del tiempo. Se dice que una serie es persistente o tiene memoria de largo plazo cuando un evento ocurrido en un instante del tiempo, tiene la capacidad de influir en los valores futuros de la serie

El análisis de persistencia de las series se hace por medio del exponente de Hurst. Existen varios métodos para calcular este exponente ${ }^{4}$; aquí se emplea el método de Re-Escalonamiento (R/S), desarrollado por Hurst (1951) que permite

2 La palabra original proviene del latín lacuna, que significa vacío. En algunos textos en castellano se ha traducido como Lagunaridad y en otros como Lacunaridad.

3 En Inglés, Box-Counting.

4 Aparte del método R/S están, Método del Espectro de Potencia, Método de Rugosidad-Longitud, Método del Variograma, y Método de Ondulaciones. 
determinar la dependencia de largo plazo de la serie. Para el cálculo del exponente ${ }^{5}$, la serie se divide en segmentos y para cada uno de ellos se calculan su media y el rango, que se normaliza por medio de la desviación estándar de los datos del segmento. Este procedimiento se hace para segmentos con diferente número de datos, sobre los que se aplica una regresión lineal,

$$
\log \left((R / S)_{N}\right)=H \log (N)+\log (c)
$$

Donde, $\mathrm{H}$ es el exponente de Hurst, $\mathrm{N}$ es el número de datos de los segmentos, y c es una constante. Los valores de $\mathrm{H}$ pueden ser:

$$
\text { Valores de } H\left\{\begin{array}{c}
0 \leq H<0,5 \\
H=0,5 \\
0,5<H \leq 1
\end{array}\right.
$$

Cuando el valor de $\mathrm{H}$ es igual o mayor que 0 y menor que 0,5 , indica que la serie es anti-persistente o ergódica ( $\mathrm{H}$ de 0 , representa un con reversión a la media y correlación negativa). Cuando el valor de $\mathrm{H}$ es igual 0,5 implica que la serie es un proceso ruido blanco. Un valor de $\mathrm{H}$ mayor de 0,5 y menor o igual a 1, es señal de que el proceso refuerza las tendencias (si hay un movimiento en un sentido durante un período, hay una alta probabilidad de que el siguiente período el movimiento sea en el mismo sentido) y tendrá correlación positiva (Gálvez, 2005).

\subsection{Caos}

La posibilidad de estructuras caóticas en los procesos se puede determinar haciendo uso del exponente de Lyapunov que es un número real asociado a la divergencia o separación de las trayectorias del sistema que se analiza en el transcurso del tiempo (Paissan, 2005); representa la separación exponencial entre dos trayectorias, inicialmente próximas al cabo de determinados pasos o iteraciones (Espinosa y Ramos, 2009) y la rapidez de difusión de los efectos de una pequeña perturbación (Montealegre, Castro y Urbano, 2002). Si se conocen las ecuaciones que definen el sistema, el exponente de Lyapunov puede calcularse de forma directa; esto no es aplicable a las series de tiempo financieras, por lo que es necesario utilizar otros algoritmos, como el desarrollado por Rosentain, Collins y De Luca (1993) .

Un sistema con uno o más exponentes de Lyapunov positivos está relacionado con un sistema caótico, y la magnitud del exponente determina la escala de tiempo

\footnotetext{
5 Este procedimiento se describe con detalle en múltiples publicaciones para citar solo algunas, Véanse León y Reveiz (2010); Gálvez (2005), Peters (1991).

6 Para el cálculo del exponente de Lyapunov en la serie de tiempo, existen en la literatura otros algoritmos como el propuesto por Wolf y otros (1985).
} 
en la que el sistema es impredecible. Un sistema dinámico sin un punto fijo tiene al menos un exponente con valor cero, que corresponde a los pequeños cambios de magnitud del eje principal tangente al flujo. Los ejes que en promedio se expanden corresponden a exponentes positivos, y viceversa. La existencia de exponentes positivos es incompatible con movimientos sujetos a atractores, a menos que emerjan trayectorias ampliamente separadas. Se define un atractor como extraño o caótico cuando un sistema se disipa con uno o más exponentes de Lyapunov positivos.

El número de exponentes de Lyapunov está determinado por la dimensión del mapa en la que está el sistema. En los mapas de una dimensión, un exponente positivo es caos, un exponente cero es un sistema de órbita marginalmente estable, y un exponente negativo es un sistema de órbita periódica.

Otros elementos que ayudan al estudio de las series bajo el enfoque de la teoría del caos se relacionan con la determinación de la longitud del ciclo y el contraste de aleatoriedad.

\subsection{Ciclos de las series}

La estimación de los ciclos de las series se hace con base en la metodología propuesta por Peters (1991), que propone dividir la serie en grupos de igual número de datos, a cada uno de ellos calcularle el exponente de Hurst y obtener su promedio. El número mínimo de grupos no debe ser menor que diez. El valor del ciclo está determinado por el período donde se ubica el mayor valor del Exponente de Hurst.

\subsection{Aleatoriedad de las series}

Para validar si la serie es aleatoria se requiere calcular el exponente de Hurst para series generadas de manera aleatoria a partir de la serie original y si este exponente se encuentra en una vecindad de 0.5 existe evidencia acerca de la aleatoriedad de la serie.

\section{DATOS}

La información que se utilizan corresponde a las variables Índice General de Valores de la Bolsa de Colombia y los rendimientos para el período comprendido entre la fusión de las tres bolsas de valores que existían en Colombia en el año 2001 (Bolsa de Valores de Bogotá, Bolsa de Valores de Medellín y Bolsa de Valores de Cali) cuando se crea el Índice General de la Bolsa de Valores de Colombia (IGBC) y la creación de lo que se ha denominado Mercado Integrado Latinoamericano (MILA) en junio de 2011 que consiste en la integración de los mercados bursátiles de Colombia, Chile y Perú. 
La serie de datos se tomó de las bases de Bloomberg y corresponde a 2.400 observaciones de los valores de cierre de las operaciones bursátiles del día, para el período comprendido entre el 30 de julio de 2001 y el 31 de mayo de 2011 sin tener en cuenta los días sábado, domingo o festivos ${ }^{7}$.

Los rendimientos del índice se calcularon como el logaritmo natural del cociente entre el valor del índice para el día y el valor del índice para el día anterior. Para los análisis de rangos re-escalonados resulta más apropiado utilizar los rendimientos logarítmicos que los rendimientos porcentuales, ya que los primeros hacen una acumulación de la suma de los retornos (Peters, 1991).

Con los valores diarios originales de cada una de las series se estimaron los promedios mensuales, trimestrales, semestrales, y anuales; y se construyeron nuevas series de datos para utilizar en los procedimientos que requiere el estudio.

En los gráficos 1 y 2 se muestra la dinámica generada por las series del IGBC y de los respectivos rendimientos y no se realiza ningún tipo de análisis estadístico de la series, ya que la metodología propuesta no los requiere.

\section{Gráfico 1. Serie de tiempo IGBC}

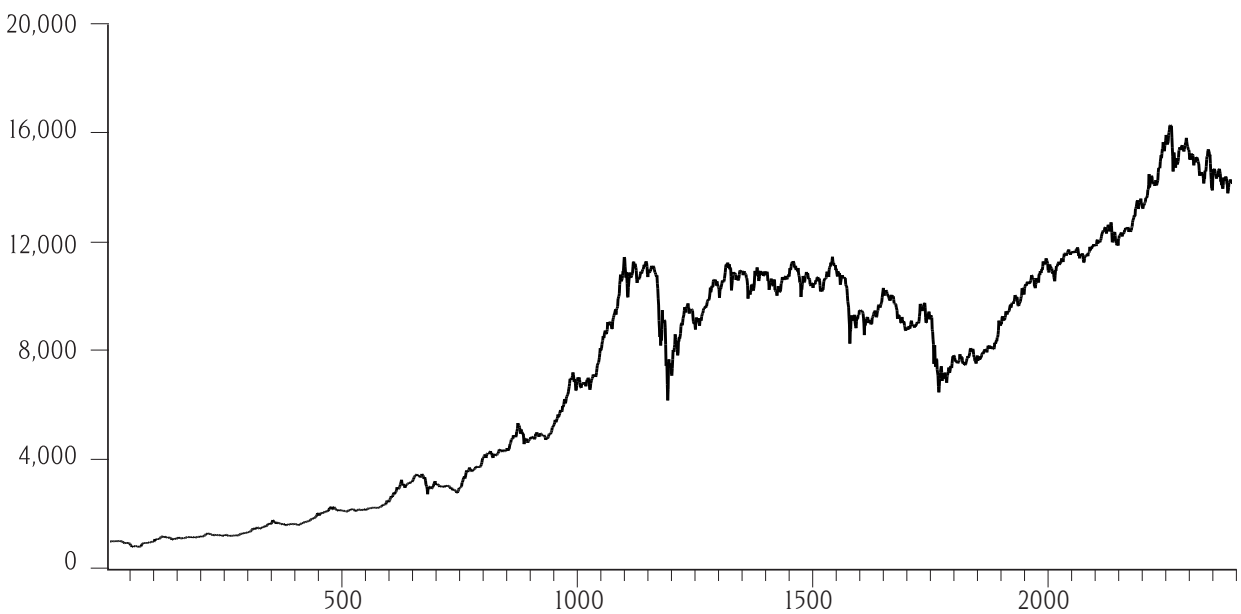

Fuente: elaboración propia, utilizando información de Bloomberg

La información utilizada en las estimaciones de este artículo se puede solicitar a través del correo electrónico: evelas@eafit.edu.co. 
Gráfico 2. Serie de tiempo para los rendimientos del IGBC

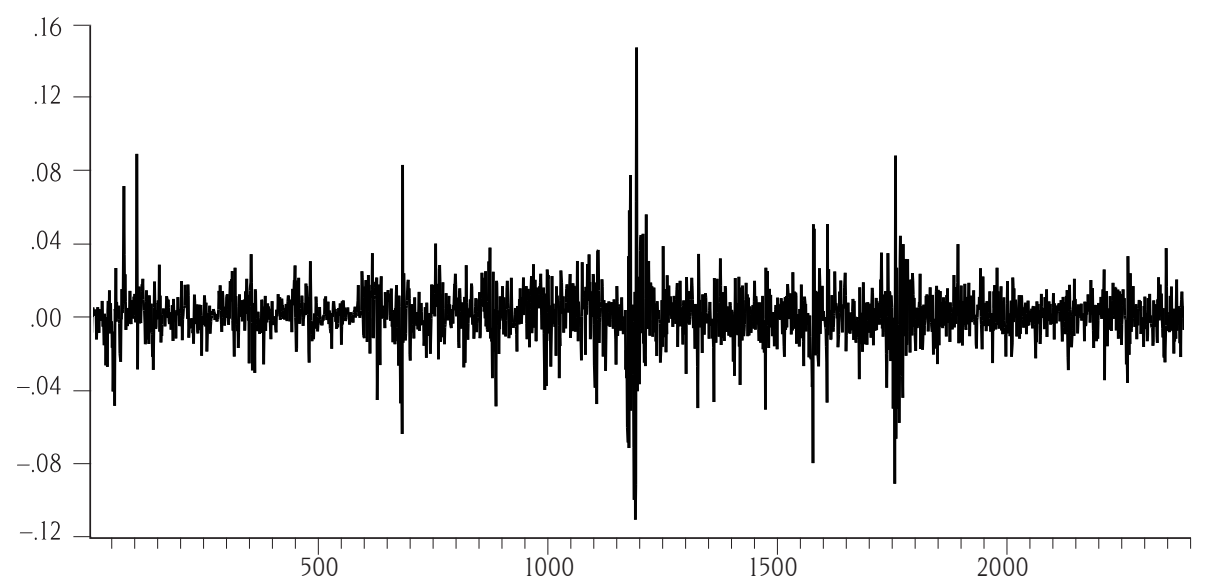

Fuente: elaboración propia, utilizando información de Bloomberg.

\section{RESULTADOS}

\subsection{Normalidad}

Para contrastar la hipótesis de normalidad se utilizan las pruebas de ShapiroWilk, Shapiro- Francia y Jarque-Bera. En la tabla 1 se reportan los valores P de las pruebas a partir de los cuales con un nivel de confianza superior al 95\% los datos presentan evidencia de no-normalidad. En el anexo se presentan las salidas obtenidas en Stata 12

Tabla 1. Valores p de los estadísticos para las pruebas de normalidad

\begin{tabular}{|l|c|c|}
\hline \multicolumn{1}{|c|}{ Test } & Valores P IGBC & Rend-IGBC \\
\hline Shapiro-Wilk & 0 & 0 \\
Shapiro-Francia & 0.0001 & 0.0001 \\
Jarque-Bera & 0 & 0 \\
\hline Jarque-Bera & 0 & 0 \\
\hline
\end{tabular}

Fuente: Elaboración propia, utilizando información de Bloomberg

\subsection{No-Linealidad}

Estudios realizados en diferentes países han mostrado la no linealidad para series similares. En este artículo se utiliza el test BDS para contrastar linealidad 
Análisis del índice general de la bolsa de valores de Colombia y sus rendimientos desde la teoría del caos, 2001-2011

frente a cualquier alternativa de no linealidad y un test para contrastar la existencia de efectos ARCH después de filtrar las series con un modelo ARIMA(p,d,q). En las tablas 2, 3 y 4 aparecen los valores P de los estadísticos utilizados en las pruebas y permiten concluir que las series corresponden a procesos no lineales.

Tabla 2. Estadísticos y valores p de la prueba BDS para el IGBC

\begin{tabular}{|c|c|c|c|c|c|c|c|}
\hline \multirow{2}{*}{ Índice } & \multirow{2}{*}{$\varepsilon / \sigma$} & \multicolumn{2}{|c|}{ Dim=2 } & \multicolumn{2}{c|}{ Dim=3 } & \multicolumn{2}{c|}{ Dim=4 } \\
\cline { 3 - 8 } & & $z$-Statistic & Prob. & $z$-Statistic & Prob. & z-Statistic & Prob. \\
\hline IGBC & $0.5 / \sigma$ & 30.7654 & 0.000 & 45.9167 & 0.000 & 66.5540 & 0.000 \\
\hline & $1 / \sigma$ & 25.9871 & 0.000 & 30.6257 & 0.000 & 34.1183 & 0.000 \\
\hline & $1.5 / \sigma$ & 23.1605 & 0.000 & 25.1182 & 0.000 & 25.8952 & 0.000 \\
\hline & $2 / \sigma$ & 21.7200 & 0.000 & 22.5134 & 0.000 & 22.3886 & 0.000 \\
\hline
\end{tabular}

Fuente: Elaboración propia, utilizando información de Bloomberg

Tabla 3. Estadísticos y valores $p$ de la prueba BDS para los rendimientos del IGBC

\begin{tabular}{|l|l|c|c|c|c|c|c|}
\hline \multirow{2}{*}{ Índice } & \multirow{2}{*}{$\varepsilon / \sigma$} & \multicolumn{2}{|c|}{ Dim=2 } & \multicolumn{2}{c|}{ Dim=3 } & \multicolumn{2}{c|}{ Dim=4 } \\
\cline { 3 - 8 } & & $z$-Statistic & Prob. & $z$-Statistic & Prob. & $z$-Statistic & Prob. \\
\hline IGBC & $0.5 / \sigma$ & 15.9749 & 0.000 & 19.6936 & 0.000 & 22.7084 & 0.000 \\
\hline & $1 / \sigma$ & 21.8150 & 0.000 & 23.2317 & 0.000 & 23.2162 & 0.000 \\
\hline & $1.5 / \sigma$ & 19.5107 & 0.000 & 21.4614 & 0.000 & 22.1460 & 0.000 \\
\hline & $2 / \sigma$ & 21.8150 & 0.000 & 23.2317 & 0.000 & 23.2162 & 0.000 \\
\hline
\end{tabular}

Fuente: Elaboración propia, utilizando información de Bloomberg

Tabla 4. Estadísticos y valores p de la prueba ARCH para el IGBC

\begin{tabular}{|l|c|c|}
\hline \multicolumn{3}{|c|}{ Test: ARCH } \\
\hline Estadístico & Valor & Valores P \\
F-statistic & 649.5164 & 0.0000 \\
Obs*R-squared & 511.3754 & 0.0000 \\
\hline
\end{tabular}

Fuente: Elaboración propia, utilizando información de Bloomberg

Tabla 5. Estadísticos y valores $\mathrm{p}$ de la prueba ARCH para los rendimientos del IGBC

\begin{tabular}{|l|c|c|}
\hline \multicolumn{3}{|c|}{ Test: ARCH } \\
\hline Estadístico & Valor & Valores P \\
\hline F-statistic & 614.5186 & 0.0000 \\
\hline Obs*R-squared & 489.4890 & 0.0000 \\
\hline
\end{tabular}

Fuente: Elaboración propia, utilizando información de Bloomberg 
Los contrastes realizados evidencian que los procesos son no lineales y no normales, condiciones necesarias para la existencia de una dinámica caótica.

\subsection{Autosimilitud}

Grafico 3. La serie IGBC en diferentes frecuencias de tiempo

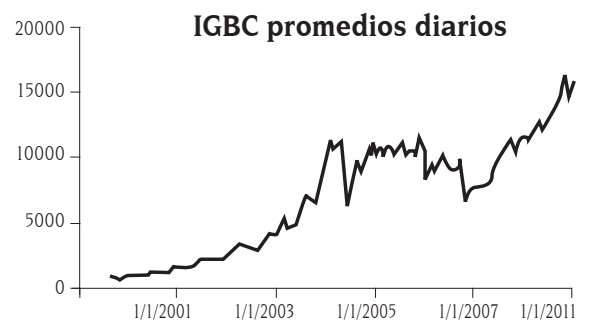

IGBC promedios mensuales

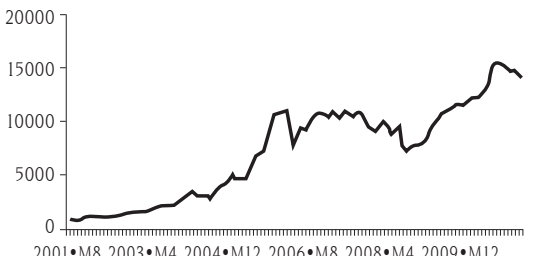

$2001 \bullet$ M8 2003•M4 2004•M12 2006•M8 2008•M4 2009•M12

IGBC promedios trimestrales

IGBC promedios semestrales
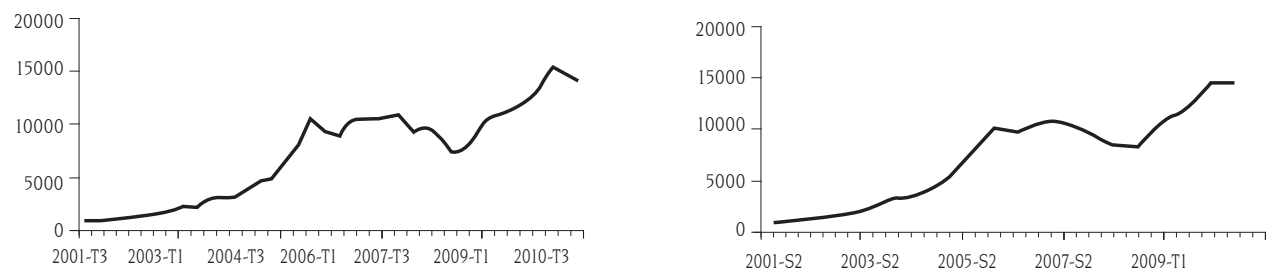

IGBC promedios anuales

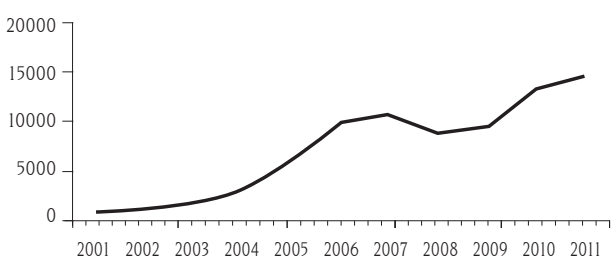

Fuente: Elaboración propia, utilizando información de Bloomberg

En el gráfico 3 aparecen las series para el IGBC con datos diarios, mensuales, trimestrales, semestrales y anuales, respectivamente. Podemos notar patrones similares a la serie diaria, lo cual constituye una primera aproximación al fenómeno de autosimilitud. Para los rendimientos del IGBC, se encuentran resultados similares. 
Para completar el estudio de la autosimilitud se utilizan la dimensión fractal y la dimensión de la lagunaridad que son los indicadores de autosimilitud estadística.

\subsection{Dimensión fractal y dimensión de lagunaridad}

La información relacionada con la dimensión fractal de la serie del IGBC y de la serie de rendimientos en las frecuencias diaria (D), mensual (M), trimestrales (T), semestral (S) y anual (A) calculada por medio de la metodología Box-Counting aparece en la tabla 6. La propiedad de auto similitud estadística para la serie de del IGBC y para los rendimientos del IGBC en las diferentes escalas analizadas se cumple, ya que los valores de la dimensión fractal en las diferentes frecuencias pertenecen a una misma vecindad.

Para las series del IGBC y rendimientos los valores para la dimensión de lagunaridad se muestran en la columna derecha de cada parte de la tabla 6, se observa que en las diferentes escalas de tiempo analizadas la dimensión fractal toma sus valores en una misma vecindad y, por tanto, podemos afirmar que existe autosimilitud.

Tabla 6. Dimensión fractal y dimensión de lagunaridad para las series IGBC y rendimiento del IGBC

\begin{tabular}{|c|c|c|c|}
\hline Serie & DF Prom & DF desv & DF Lac \\
\hline IGBC-D & 1.78 & 0.6196 & 0.1206 \\
\hline IGBC-M & 1.8 & 0.5874 & 0.1071 \\
\hline IGBC-T & 1.81 & 0.5484 & 0.0916 \\
\hline IGBC-S & 1.81 & 0.5329 & 0.0859 \\
\hline IGBC-A & 1.83 & 0.5097 & 0.0778 \\
\hline
\end{tabular}

\begin{tabular}{|c|c|c|c|}
\hline Serie & DF Prom & DF desv & DF Lac \\
\hline Rend-IGBC-D & 1.6 & 0.8987 & 0.3167 \\
\hline Rend-IGBC-M & 1.52 & 0.9708 & 0.3096 \\
\hline Rend-IGBC-T & 1.66 & 0.8192 & 0.244 \\
\hline Rend-IGBC-S & 1.73 & 0.7176 & 0.173 \\
\hline Rend-IGBC-A & 1.73 & 0.7143 & 0.171 \\
\hline
\end{tabular}

Fuente: Elaboración propia, utilizando información de Bloomberg

\subsection{Persistencia}

En la tabla 7 aparecen los reportes asociados con el cálculo del exponente de Hurts para la serie original y para las series en diferentes escalas de tiempo (mensual, trimestral, semestral y anual). Estos resultados permiten afirmar que las dos series son persistentes, es decir, que tienen memoria. 
Hermilson Velásquez Ceballos - Jorge Humberto Restrepo Restrepo

Tabla 7. Exponente de Hurst para las series del IGBC y rendimientos

\begin{tabular}{|c|c|c|}
\hline Serie & Exp.Hurst & Datos \\
\hline IGBC-D & 1.0 & 2400 \\
\hline IGBC-M & 1.0 & 118 \\
\hline IGBC-T & 1.0 & 40 \\
\hline IGBC-S & N/D & 20 \\
\hline IGBC-A & N/D & 11 \\
\hline
\end{tabular}

\begin{tabular}{|c|c|c|}
\hline Serie & Exp. Hurst & Datos \\
\hline Rend-IGBC-D & 0.61 & 2400 \\
\hline Rend-IGBC-M & 0.70 & 118 \\
\hline Rend-IGBC-T & 0.70 & 40 \\
\hline Rend-IGBC-S & N/D & 20 \\
\hline Rend-IGBC-A & N/D & 11 \\
\hline
\end{tabular}

Fuente: Elaboración propia, utilizando información de Bloomberg

\subsection{Caos}

La información relacionada con el exponente de Lyapunov para la serie del IGBC y la de los rendimientos aparece en la tabla 7. Estos cálculos se obtienen al utilizar el software MTRCHAOS, cuyo fundamento formal fue publicado por Rosentain, Collins y De Luca (1993). En la tabla se puede observar que todos los valores del exponente de Lyapunov son positivos en las tres dimensiones analizadas; esto permite concluir que la serie del IGBC tiene un comportamiento caótico. Para la serie de los rendimientos del IGBC, aunque existen algunos exponentes con valores negativos, puede afirmarse que la serie sigue dinámica caótica, porque el máximo exponente es positivo, condición necesaria y suficiente.

Tabla 8. Exponente de Lyapunov de los Rendimientos del IGBC para las dimensiones 2, 3 y 4

\begin{tabular}{|c|c|c|c|c|c|c|}
\hline \multirow{2}{*}{} & \multicolumn{3}{|c|}{ IGBC } & \multicolumn{3}{c|}{ Rend-IGBC } \\
\cline { 2 - 7 } & Dim=2 & Dim=3 & Dim=4 & Dim=2 & Dim=3 & Dim=4 \\
\hline 0 & 0.1531 & 0.1335 & 0.1251 & 0.1206 & 0.0987 & 0.0321 \\
\hline 1 & 0.1061 & 0.1043 & 0.1035 & 0.0258 & 0.0391 & 0.3040 \\
\hline 2 & 0.0809 & 0.0819 & 0.0879 & 0.0023 & 0.0140 & 0.2149 \\
\hline 3 & 0.0708 & 0.0664 & 0.0734 & 0.0018 & 0.0020 & 0.2152 \\
\hline 4 & 0.0655 & 0.0581 & 0.0626 & 0.0026 & 0.0005 & -0.0545 \\
\hline 5 & 0.0618 & 0.0528 & 0.0567 & 0.0039 & -0.0002 & -0.1357 \\
\hline
\end{tabular}

Fuente: elaboración propia, utilizando información de Bloomberg

\subsection{Aleatoriedad}

En la tabla se muestra el exponente de Hurst para organizaciones aleatorias y podemos notar que para serie del IGBC se alcanza el valor 0.5 en solo un ensayo; 
para la serie de rendimientos, al aumentar el número de ensayos, la serie converge a 0.5 , resultados que nos indican aleatoriedad en las series analizadas.

Tabla 9. Exponente de Hurst para las series del IGBC y rendimientos después de ordenarlas de manera aleatoria.

\begin{tabular}{|c|c|c|c|c|c|c|c|}
\hline \multirow{2}{*}{ Índice } & \multirow{2}{*}{ Datos } & \multirow{2}{*}{ Exp. Hurst } & \multicolumn{5}{|c|}{ Organización aleatoria } \\
\cline { 4 - 8 } & & & 1 & 2 & 3 & 4 & 5 \\
\hline IGBC & 2400 & 1.0 & 0.50 & 0.49 & 0.57 & 0.53 & 0.63 \\
\hline Rend-IGBC & 2400 & 0.61 & 0.64 & 0.59 & 0.53 & 0.54 & 0.55 \\
\hline
\end{tabular}

Fuente: Elaboración propia, utilizando información de Bloomberg

\subsection{Ciclos de las series}

El cálculo de los ciclos de las series analizadas se hizo con base en la metodología propuesta por Peters (1991). La serie del IGBC original de 2.400 datos se fraccionó en segmentos de $\mathrm{N}$ datos, de manera inicial con 80 datos e incrementando en 20 observaciones en cada una de las muestras hasta llegar a 240. A cada sub-muestra de la serie fraccionada se le calculó el exponente de Hurst y sobre estos se obtuvo un promedio para las diferentes series de N datos. Un procedimiento similar se realizó con la serie de los rendimientos del IGBC. Los resultados se muestran en la tabla 10. El ciclo de la serie se determina con el valor de $\mathrm{N}$ que corresponda al mayor valor del exponente de Hurst. Para la serie del IGBC corresponde a 180 días bursátiles y para la serie de los rendimientos del IGBC corresponde a 80 días bursátiles.

Tabla 10. Valores promedio del Exponente de Hurst para las series Fragmentadas del IGBC y Rendimientos.

$$
\mathrm{N}=\# \text { de datos. }
$$

\begin{tabular}{|c|c|c|}
\hline $\mathrm{N}$ & IGBC & Rend-IGBC \\
\hline 80 & 1.011 & 0.722 \\
\hline 100 & 1.018 & 0.697 \\
\hline 120 & 1.002 & 0.689 \\
\hline 140 & 1.021 & 0.688 \\
\hline 160 & 1.011 & 0.682 \\
\hline 180 & 1.032 & 0.675 \\
\hline 200 & 1.016 & 0.671 \\
\hline 220 & 1.015 & 0.641 \\
\hline 240 & 1.01 & 0.653 \\
\hline
\end{tabular}

Fuente: Elaboración propia, utilizando información de Bloomberg 


\section{CONCLUSIONES}

El análisis que se realizó bajo este enfoque evidencia la no linealidad y la ausencia de normalidad en las series objeto de estudio, resultados que deben considerar aquellas metodologías que parten del supuesto de normalidad para construir sus desarrollos, recomendación similar para cuando se utilizan procesos lineales.

Las propiedades de autosimilitud, persistencia y caos, unidas a la no-linealidad y ausencia de normalidad, permiten aprovechar las propiedades de los objetos fractales y de la teoría del caos, para incorporar nuevas herramientas de análisis en los mercados financieros, las cuales abren la posibilidad de diseñar estrategias alternativas de negociación, que podrían ser aprovechadas en procesos de transacción en las bolsas de valores.

Los hallazgos de esta investigación se suman a otros similares con índices financieros que aportan evidencia de estructuras caóticas, y no comportamientos aleatorios como lo sugieren otras teorías, resultados que muestran la urgencia de construir nuevos procedimiento de análisis para estudiar la dinámica del mercado de capitales, que por su naturaleza es altamente compleja, dado que pueden coexistir procesos estocásticos, caos y fractales. Bajo el supuesto de caos para el mercado, es posible considerar procesos no-lineales entre los cuales se tienen las redes neuronales para capturar la dinámica.

Estos resultados plantean nuevos retos en la búsqueda de la explicación de la dinámica del mercado, así como la necesidad de considerar otras alternativas, que puedan ser utilizadas en el diseño de estrategias de negociación, o combinar diferentes como puede ser caos-cointegración.

\section{BIBLIOGRAFIA}

Bartolozzi, M.; Drozdz, S.; Leinweber, D. B.; Speth, J. y Thomas, A. W. (2005). Self-Similar LogPeriodic Structures in Western Stock Markets from 2000. En: International Journal of Modern Physics C, Vol. 16, No. 9, p. 1347-1361.

Brock, W. A.; Dechert, W. D.; Scheinkman, J. y LeBaron, B. (1996). A test for independence based on the correlation dimension. En: Econometric Reviews. Vol. 15, No. 3, p. 197-235.

Casparri, Maria Teresa y Moreno, Alejandro (2008). Geometría Fractal y Mercados Financieros. [En línea] Actas de las XIII Jornadas de Epistemología de las Ciencias Económicas. Centro de Investigación en Métodos Cuantitativos Aplicados a la Economía y a la Gestión. Universidad de Buenos Aires. http://www.econ.uba.ar/www/institutos/epistemologia/marco _archivos/ ponencias/Actas\%20XIII/Trabajos\%20Episte/Casparri-Moreno _trabajo.pdf. Fecha de consulta: 01.07.2011. 
Espinosa Méndez, Christian (2009). Comportamiento Caotico en los Mercados Bursátiles Latinoamericanos Utilizando Visual Recurrence Analysis. En: Análisis Económico, Vol. XXIII, No. 52, enero-abril, p. 159-183.

Fama, Eugene F.; Fisher, Lawrence; Jensen, Michael C. y Roll, Richard (1969). The adjustment of stock prices to new information. En: International Economic Review, Vol. 10, No. 1, febrero, p. $1-21$.

Gálvez Medina, Ernesto Teobaldo. (2005). Análisis Fractal del Mercado de Valores de México (19782004). Tesis Doctoral. [En línea] Escuela Superior de Comercio y Administración, Sección de Estudio de Postgrado e Investigación, Unidad Santo Tomás, Instituto Politécnico Nacional. México D. F. http://www.colparmex.org/Tesis/EGM.pdf. Fecha de Consulta: 01.02.2011.

Hurst, H. E. (1951). The Long-termstorage capacity of reservoirs. Transactions of the American Society of Civil Engineers, Vol. 116, p. 770-808.

León, Carlos; Reveíz, Alejandro (2010). Portafolio Organization and Long-Term Dependance. [En lineal Borradores de Economía Banco de la República. No 622. http://www.banrep.gov.co/ docum/ftp/borra622.pdf. Fecha de consulta: 21.01.2011.

Mandelbrot, B. (1983). The Fractal Geometry of Nature. New York: W.H. Freeman and Company, 468p.

Montealegre, C. R.; Castro, I. y Urbano, F. (2002). Minimal Lagrangian submanifold in the complex hyperbolic space, Illinois J. Math, Vol. 46, No. 3 (otoño), p. 695-721

Nawrocki, David. (1995). R/S Analysis and Long Term Dependence in Stock Market Indices. En: Managerial Finance, Vol. 21, No. 7, p. 78-91.

Özer, Göklan; Ertokatli, Cengiz. (2010). Chaotic Processes of Common Stock Index Returns: An Empirical Examination of Istanbul Stock Exchange (ISE) Market. En: African Journal of Business Management. Vol. 4, No. 6, junio, p. 1140-1148.

Ozun, Alper; Hanias, Mike P.; Curtis, Panayoitis G. (2010). A Chaos Analysis for Greek and Turkish Equity Markets. En: EuroMed Journal of Business, Vol. 5 No. 1, p. 101-118.

Paissan, Gabriel (2005). Métodos de Análisis de Datos Caóticos. [En línea] http://dc222.4shared. com/doc/d8dDrPBp/preview.html . Fecha de consulta: 21.01.2011.

Peters, Edgar E. (1989). Fractal Structure in the Capital Markets. En: Financial Analysts Journal, Vol. 45, No. 4, julio-agosto, p. 32-37.

Peters, Edgar E. (1991). Chaos and Order in the Capital Markets: A New View of Cycles, Prices and Markey Volatility. Nueva York, John Wiley E Sons, Inc., 292p.

Restrepo Restrepo, Jorge Humberto. (2011). Análisis del índice general de las bolsas de valores de Colombia (IGBC), chile (IPSA) y Perú (IGBVL), y sus rendimientos desde la teoría del caos 2001-2011, Medellín, Tesis Maestría en Finanzas.

Rosenstein, Michael; Collins, J. James y De luca, Carlo. (1993). A practical method for calculating largest Lyapunov Exponents from small data sets. En: Physica D. Vol. 65, p. 117-134.

Wolf, A.; Swift, J.; Swinney, H.; y Vastano, J. (1985). Determining lyapunov exponents from a time series. Physica D. Vol. 16, p. 285-317. 


\section{ANEXOS}

\section{ANEXO A: REPORTE DE E-VIEWS 6.0 PARA EL CONTRASTE DE NO LINEALIDAD DE LAS VARIABLES}

\begin{tabular}{llll}
\hline \multicolumn{4}{c}{ Heteroskedasticity Test: ARCH } \\
\hline F-statistic & 649.5164 & Prob. F(1,2395) & 0.0000 \\
Obs*R-squared & 511.3754 & Prob. Chi-Square(1) & 0.0000 \\
\hline
\end{tabular}

Test Equation:

Dependent Variable: RESID^2

Method: Least Squares

Date: 29/05/12 Time: 10:16

Sample (adjusted): 42400

Included observations: 2397 after adjustments

\begin{tabular}{lcccc}
\hline & Coefficient & Std. Error & t-Statistic & Prob. \\
\hline \multicolumn{1}{c}{ C } & 7125.741 & 859.1838 & 8.293617 & 0.0000 \\
\multicolumn{1}{c}{ RESID $^{\wedge}$ 2(-1) } & 0.461883 & 0.018123 & 25.48561 & 0.0000 \\
\hline R-squared & 0.213340 & Mean dependent var & 13233.88 \\
Adjusted R-squared & 0.213011 & S.D. dependent var & 45534.98 \\
S.E. of regression & 40395.17 & Akaike info criterion & 24.05164 \\
Sum squared resid & $3.91 \mathrm{E}+12$ & Schwarz criterion & 24.05647 \\
Log likelihood & -28823.89 & Hannan-Quinn criter. & 24.05340 \\
F-statistic & 649.5164 & Durbin-Watson stat & 2.214043 \\
Prob(F-statistic) & 0.000000 & & \\
\hline \multicolumn{5}{c}{ Heteroskedasticity Test: ARCH } \\
\hline F-statistic & 614.5186 & Prob. F(1,2396) \\
Obs*R-squared & 489.4890 & Prob. Chi-Square(1) & 0.0000 \\
\hline
\end{tabular}

Test Equation:

Dependent Variable: RESID^2

Method: Least Squares

Date: 29/05/12 Time: 10:26

Sample (adjusted): 32400

Included observations: 2398 after adjustments

\begin{tabular}{lllll}
\hline & \multicolumn{1}{c}{ Coefficient } & \multicolumn{1}{c}{ Std. Error } & t-Statistic & \multicolumn{1}{c}{ Prob. } \\
\hline \multicolumn{1}{c}{ C } & 0.000111 & $1.43 \mathrm{E}-05$ & 7.759306 & 0.0000 \\
\multicolumn{1}{c}{ RESID $^{\wedge} 2(-1)$} & 0.451798 & 0.018225 & 24.78949 & 0.0000 \\
\hline R-squared & 0.204124 & & Mean dependent var & 0.000202 \\
Adjusted R-squared & 0.203792 & & S.D. dependent var & 0.000756 \\
S.E. of regression & 0.000675 & Akaike info criterion & -11.76377 \\
Sum squared resid & 0.001091 & Schwarz criterion & -11.75895 \\
Log likelihood & 14106.76 & Hannan-Quinn criter. & -11.76201 \\
F-statistic & 614.5186 & Durbin-Watson stat & 2.202074 \\
Prob(F-statistic) & 0.000000 & &
\end{tabular}




\section{ANEXO B: REPORTE DE STATA 12 PARA LOS CONTRASTES DE NORMALIDAD}

- swilk IGBC RendIGBC

Shapiro-Wilk W test for normal data

\begin{tabular}{r|cccccccc}
\multicolumn{1}{l|}{ Variable } & Obs & & W & & V & $z$ & Prob $>z$ \\
\hline IGBC & 2400 & 0 & 92036 & 111 & 471 & 12 & 070 & 0.00000 \\
RendIGBC & 2400 & 0 & 88270 & 164 & 178 & 13 & 061 & 0.00000
\end{tabular}

- sfrancia IGBC RendIGBC

Shapiro-Francia W' test for normal data

\begin{tabular}{r|cccccccc}
\multicolumn{1}{l|}{ Variable } & Obs & \multicolumn{3}{c}{ W' $^{\prime}$} & & V' $^{\prime}$ & $z$ & Prob $>z$ \\
\hline IGBC & 2400 & 0. & 92103 & 117 & 170 & 11 & 535 & 0.00001 \\
RendIGBC & 2400 & 0. & 88056 & 177 & 222 & 12 & 537 & 0.00001
\end{tabular}

- jb IGBC

Jarque-Bera normality test: $163.6 \mathrm{Chi}(2)$ 3.0e-36

Jarque-Bera test for Ho: normality:

- jb RendlGBC

Jarque-Bera normality test: $1.5 \mathrm{e}+04 \mathrm{Chi}(2) 0$

Jarque-Bera test for Ho: normality: 


\section{ANEXO C: REPORTES DEL EXPONENTE DE HURST}

\section{IGBC - Frecuencia diaria}

Exponente de Hurst

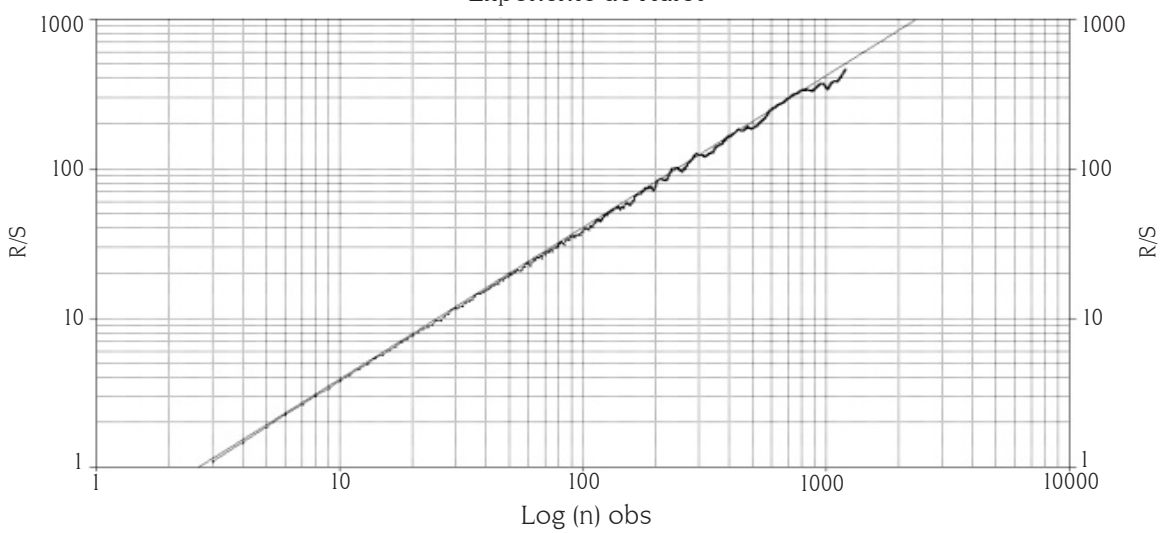

IGBC - Rendimientos diarios

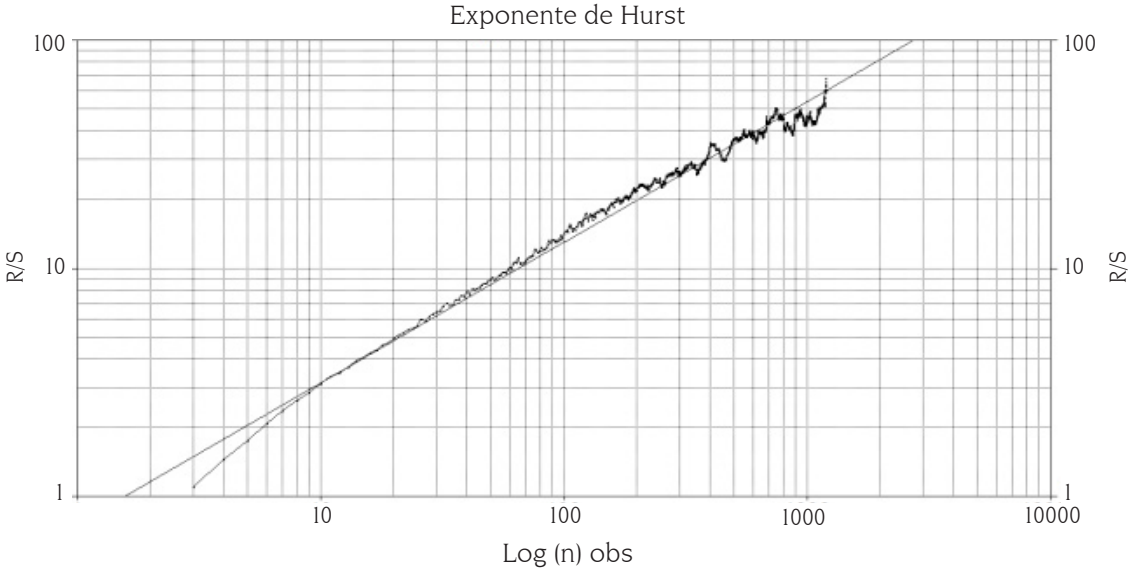

Fuente: Elaboración propia con datos de Bloomber 